\title{
Central Projection of the Peripheral Cochlear Nerve from Each Turn to the Cochlear Nuclei in the Mongolian Gerbil
}

\author{
Hong-Joon Park ${ }^{1}$ and Hee-Nam Kim ${ }^{2}$
}

\begin{abstract}
The central projections of the peripheral cochlear nerve fiber from each turn to the cochlear nuclei $(C N)$ in the mongolian gerbil were investigated using retrograde transportation of horseradish peroxidase (HRP). The organ of Corti and the osseous spiral lamina were scratched with an electrolytically-sharpened fine needle via a small hole at each turn of the cochlea. The cochlea was filled with a $30 \%$ horseradish peroxidase (HRP) solution. After 48 hours, $50 \mu \mathrm{m}$ transverse serial sections of the brainstem were made with a vibratome. The tissue was processed with the diaminobenzidine procedure of the cobalt-glucose method. Our experiment revealed that the fibers from the basal turn terminated at the dorsomedial portion of anteroventral cochlear nuclei (AVCN), but those from the apical turn were distributed among the ventrolateral portion of the AVCN. In the posteroventral cochlear nuclei (PVCN) and dorsal cochlear nuclei (DCN), the fibers from basal to apical turns extend from the dorsal to the ventral portion of each nuclei. A distinct tonotopic arrangement could be found between the origin of cochlear fibers of each turn and their termination in the regions of the cochlear nuclei (CN). Also, the results suggested that the scratch method combined with retrograde transportation of horseradish peroxidase was useful in investigating the tonotopic arrangement of the peripheral auditory nerve in the $C N$.
\end{abstract}

Key Words: Cochlea, tonotopy, HRP, gerbil

The auditory nerve fibers extend along the basilar membrane into the cochlear nuclear complex in the brainstem. It is very important to understand the precise projection patterns of this projection because the cochlear nucleus (CN) is the first relay station for central processing of auditory information. Olsen (1970) and Evans (1972) describe the tonotopic organization within the division of the $\mathrm{CN}$. They claimed that the termination of the primary cochlear nerve fibers in each $\mathrm{CN}$ occurs with low-frequency fibers projecting ventrally and

Recevied December 30, 1994

Accepted April 6, 1995

Department of Otolaryngology, Ajou University School of Medicine', Suwon 442-749, Korea

Department of Otorhinolaryngology, Yonsei University College of Medicine ${ }^{2}$ C.P.O. Box 8044, Seoul 120-752, Korea high-frequency fibers projecting dorsally. Such methods as tracer, and degenerative study are applied to investigate the projecting patterns of the $\mathrm{CN}$ in various animals. Jones \& Casseday (1978) used autoradiography to describe the general patterns of the peripheral auditory nerve projection into the cochlear nucleus in cat by injecting tritiated amino acids into the cochlea.

In the 1980's, Martin et al injected HRP into the spiral ganglion cell and traced the projection of peripheral auditory fibers into the cochlear nucleus. This team also found a tonotopic organization for high to low chracteristic frequencies for dorsoventral direction. In this study, the authors investigated the projecting patterns of each cochlear turn into the cochlear nuclei using retrograde transportation of horseradish peroxidase 
(HRP), and analyzed the distribution of primary cochlear nerve fibers in each cochlear nucleus in the mongolian gerbil.

\section{MATERIALS AND METHODS}

Twenty ears from healthy adult mongolian gerbils each weighing $65 \sim 70 \mathrm{~g}$ were used in this study. The animals were anesthetized with an intramuscular injection of xylazine $(8 \mathrm{mg} / \mathrm{kg})$ and ketamine $(40 \mathrm{mg} / \mathrm{kg})$. The bulla was exposed and opened by the postauricular approach. Each cochlear turn was identified under a Zeiss operating microscope without any damage to the stapedial artery. The ventrolateral aspect of each cochlear turn (scala tympani) was punctured using a $1 \mathrm{~mm}$ diameter diamond burr. Then, with an electrolytically-sharpened fine needle, the osseous spiral lamina and organ of Corti were scratched. By only making a small hole, we avoided scratching the cochlea for control study. The cochlea was filled with a $30 \%$ HRP solution (Sigma VI) for ten minutes and the hole was then sealed off with subcutaneous fat tissue. After 48 hours, we fixed the brainstem by intracardiac perfusion with a $200 \mathrm{cc}$ mixture solution of $2 \%$ paraformaldehyde and $0.25 \%$ glutaraldehyde. After embedding in $5 \%$ agar, we dissected the brainstem with a vibratome (Series 1000, International INC., St. Louis, USA) to make 50 rm transverse serial sections. Each section was exposed to the diaminobenzidine procedure of the cobalt-glucose oxidase method (Itoh et al. 1979). Toluidine blue was used to indicate counterstaining. Camera lucida drawings were made of each section and data of each individual area were analysed with a VIDS VI program.

\section{RESULTS}

Three major $\mathrm{CN}$ receive primary afferent fi-

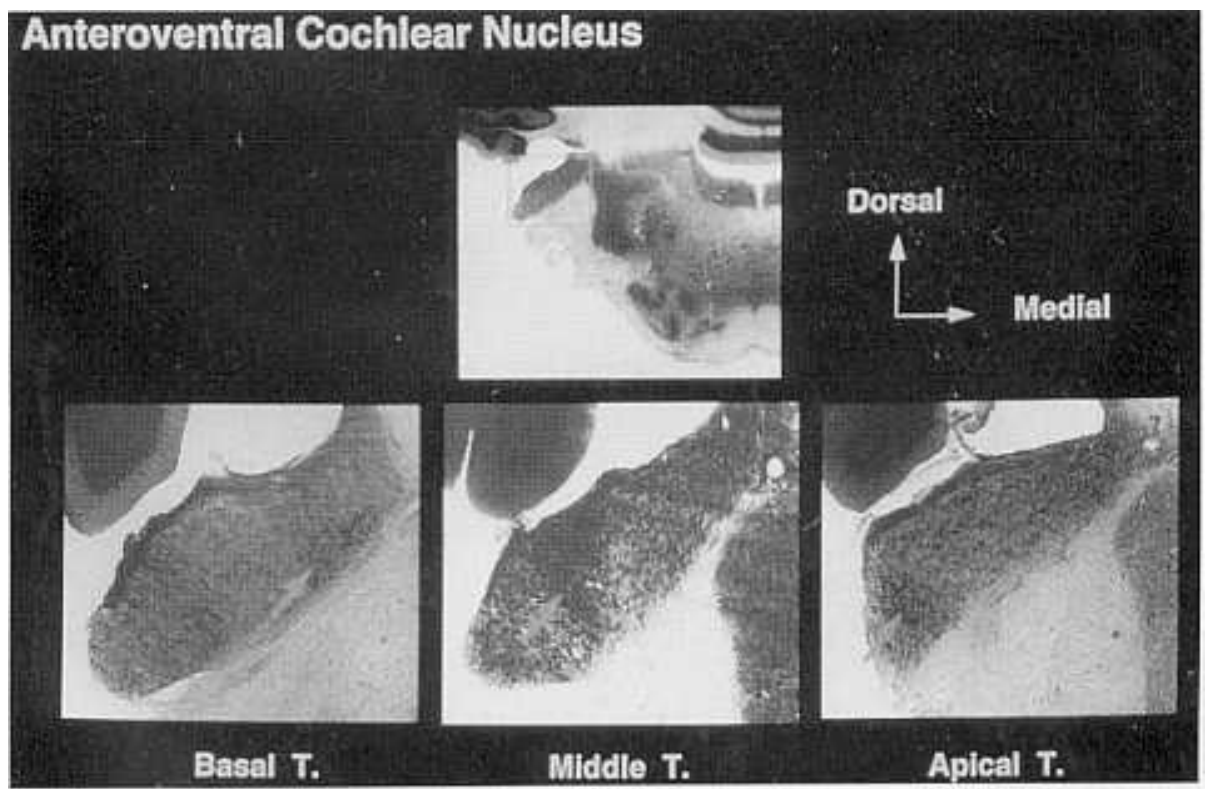

Fig. 1. Projection patterns of the primary auditory nerve fibers from each turn of the cochlea into the cochlear nuclei in the AVCN.

Above: transverse section of the brainstem; red line square indicates the area of AVCN $(\times 25)$. Below: projection of primary fibers from basal (left), middle (center) and apical turn (right) (red arrow) $(\times 100)$. 


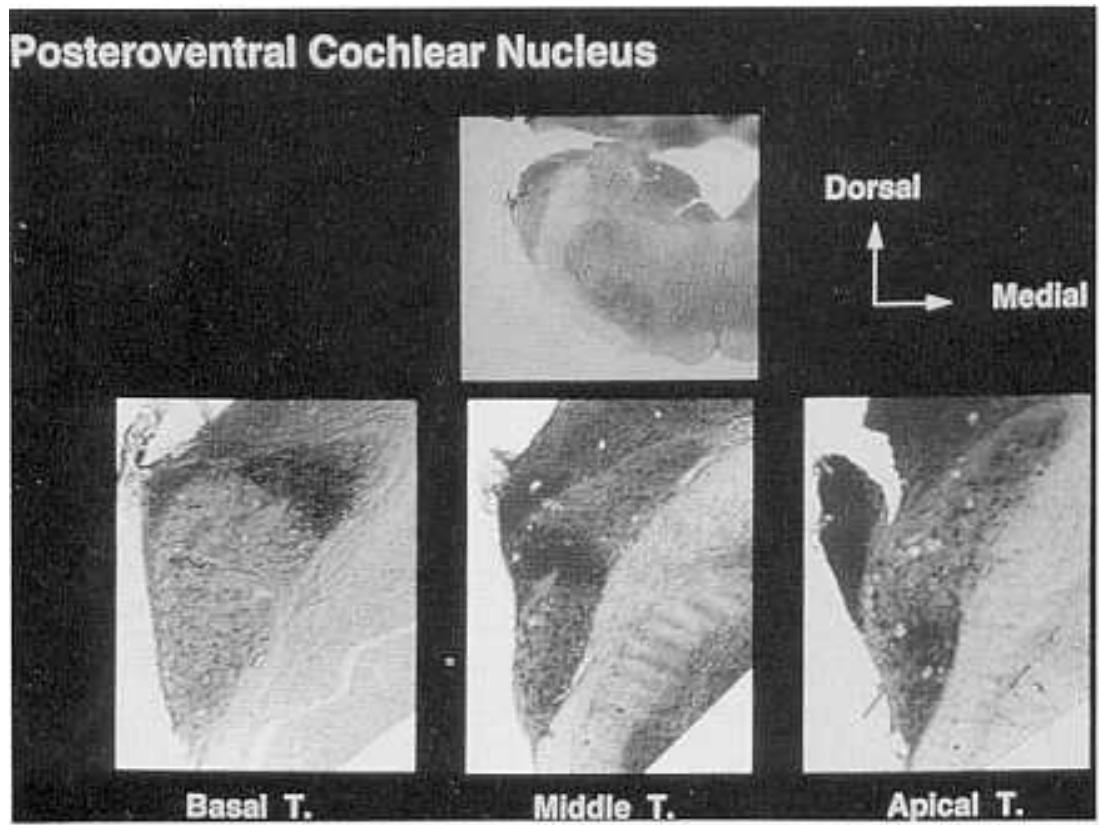

Fig. 2. Projection patterns of the primary auditory nerve fibers from each turn of the cochlea into the cochlear nuclei in the PVCN.

Above: transverse section of the brainstem; red line square indicates the area of PVCN $(\times 25)$. Below: projection of primary fibers from basal (left), middle (center) and apical turn (right) (red arrow) $(\times 100)$.

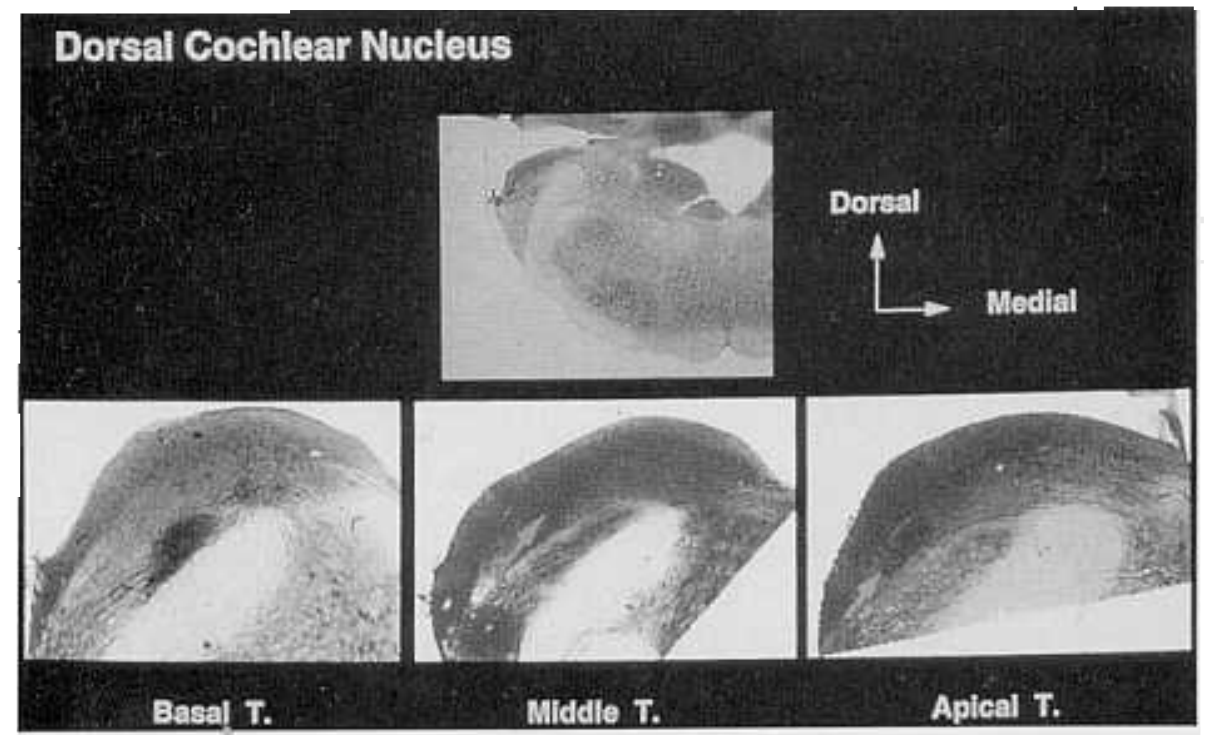

Fig. 3. Projection patterns of the primary auditory nerve fibers from each turn of the cochlea into the cochlear nuclei in the DCN.

Above: transverse section of the brainstem; red line square indicates the area of $D C N(\times 25)$. Below: projection of primary fibers from basal (left), middle (center) and apical turn (right) (red arrow) $(\times 100)$. 
Table 1. Proportion of primary cochlear nerve fibers in each $\mathrm{CN}$ from each turn of the cochlea (\%)

\begin{tabular}{lccc}
\hline \hline & Apical turn & Middle turn & Basal turn \\
\hline AVCN & 46.7 & 46.0 & 47.8 \\
PVCN & 34.9 & 34.1 & 40.0 \\
DCN & 18.4 & 19.9 & 12.2 \\
\hline
\end{tabular}

bers at each turn of the cochlea. The primary cochlear nerve fibers from the basal turn of the cochlea are projected into the dorsomedial region in a band-like pattern, while those from the apical turn are projected into ventrolateral region in the AVCN. The intermediate region is innervated by fibers from the middle turn (Fig 1).

The neurons from the dorsal portion of the PVCN are synapsed with fibers from the basal turn, while those of the ventral portion are synapsed with fibers from the apical turn. Fibers from the middle turn are distributed to the intermediate region (Fig 2).

In the $\mathrm{DCN}$, the primary cochlear nerve fibers from the basal turn of the cochlea projected into the dorsomedial region, but the fibers from the apical turn projected into the ventrolateral region. The fibers from the middle turn projected into the intermediate region between the basal and apical turns (Fig 3).

The proportion of primary cochlear nerve fibers in each CN was calculated by distribution area. Most of the fibers from each turn were distributed to the AVCN and the PVCN. Only $10 \sim 20 \%$ fibers were distributed to the DCN (Table 1).

\section{DISCUSSION}

There have been numerous reports regarding the central projection of the cochlear nerve since Lorente de No (1933) described the arrival of the cochlear fiber in the medulla within the anatomy of the eight nerve in rabbit. He observed that the highest point of the ventral nucleus corresponds to the apical region and that the lowest point to the basilar part of the ganglion. Culler (1935) and Stevens (1935) also noted the tonotopic arrangement of the organ of Corti. Compared to previous studies, the topographic projection of the cochlear nerve fibers into the cochlear nuclei in mammalian animals was consistent. The fibers from the basal turn of cochlea terminate dorsomedially and those of the apical turn, ventrolaterally in the cochlear nuclear complex (Rasmussen et al. 1960; Sando 1965; Van Noort 1969). These results were quite similar to our results.

The gerbil was chosen as the experimental animal because its large bulla allows for easy access to the cochlea (Lay 1972), along with the fact that it has a similar low frequency threshold as humans (Ryan 1976). Their auditory function may be more encephalized relative to other rodents (Frisina et al. 1982).

In general, the experimental modes used to analyze the central projection of the peripheral nerve were degeneration, and tracer methods. There are several cochlear projection studies using the degenerative method (Lewy and Kobrak 1936; Webster 1971; Moskowitz \& Liu 1972; Noda \& Pirsig 1974). Unfortunately, the process requires time to induce degenerative changes on the nervous system. Among tracer methods, HRP is rapidly absorbed by nerve terminals, as well as by axons at the site of axonal injury, and is transported in a retrograde direction (Aldskogius. 1983). It is easy to use, and 48 hours is sufficient for it to transport into the gerbil's auditory system.

Lee et al. (1989) used the scratch method to label HRP in the vestibular system. The scratch was made by with an eletrolyticallysharpened fine needle in the vestibular sensory epithelium. With this technique, it was possible to distinguish the central projection from the central and peripheral portion of the crista ampullaris in the lateral canal (Park et al. 1992). Application of the scratch technique made it possible to make a precise lesion on each turn of the cochlea.

Over $80 \%$ of primary cochlear fibers from each turn projected into the VCN; in contrast, less than $20 \%$ of the fibers projected into the DCN. Frisina et al. (1982) recorded the fiber's 
volume ratio in the $\mathrm{VCN}$ and $\mathrm{DCN}$. The report noted that the relative size of the DCN decreases with phylogenetic development. Furthermore, they calculated that in the gerbil, the ratio is 3.9. This is consistent with our results. The possible significance of these results was that for lower mammals, relatively high-level processing takes place in the $\mathrm{DCN}$, whereas in higher mammals, the DCN may not be involved in high-level processing. Rather, this process may take place in the auditory centers of the brainstem in the higher mammals (Osen. 1969).

In summary, we confirmed that our results were consistent with those for other mammalian species. A distinct tonotopic arrangement between the origin of cochlear fibers of each turn and their termination in the regions of the $\mathrm{CN}$ were found. Moreover, we suggest that the scratch method of end-organs in combination with retrograde transportation of horseradish peroxidase was useful for investigating the tonographic arrangement of the peripheral auditory nerve in the cochlear nucleus.

\section{REFERENCES}

Aldskogius H, Arvidsson J, Erik K: Movement of horseradish peroxidase after its entry into intact and damaged peripheral nerve axon. Exp Neur 79: 862-866, 1983

Culler E: An experimental study of tonal localization in the cochlea of the guinea pig. Ann Otol (St. Lou is) 44: 807-813, 1935

Evans EF: The frequency response and other properties of single fibers of the guinea pig cochlear nerve. J Physial 226: 263-287, 1972

Frisina RD, Chamberlain SC, Brachman ML, Smith RL: Anatomy and physiology of the gerbil cochlear nucleus: An improved surgical approach for microelectrode studies. Hearing Res 6: 259-275, 1982

Itoh K, Konishi A, Nomura S, Mizuno N, Nakamura Y, Sugimoto T: Application of coupled oxidation reaction to electronmicroscopic demonstration of horseradish peroxidase: cobalt-glucose oxidase method. Brain Res 175: 341-346, 1979
Jones DR, Casseday JH: Auditory nerve: Projection from cochlea demonstrated by autoradiographic methods. Brain Res 148: 224-229, 1978

Lay D: The anatomy, physiology, functional significance and evolution of specialized hearing organs of gerbilian rodents. $J$ Morphol 138: 41120,1972

Lee WS, Newman A, Honrubia V: HRP labelling of afferent vestibular nerve fiber in mammals. Cell Biol Intern Reports 13: 635-636, 1989

Lewy FH, Kobrak H: The neural projection of the cochlear spirals on the primary acoustic centers. Arch Neurd Psychiat (Chic) 35: 839-852, 1936

Lorente de No R: Anatomy of the eighth nerve. Laryngoscope 43: 1-38, 1933

Moskowitz N, Liu J: Central projection of the spiral ganglion of the squirrel monkey. J Comp Neurol 144: 335-344, 1972

Noda Y, Pirsig W: Anatomical projection of the cochlea to the cochlear nuclei of the guinea pig. Arch Otorhindaryng 208: 107-120, 1974

Osen KK: Cytoarchitecture of the cochlear nuclei in the cat. J Comp Neur 139: 453-484, 1969

Osen KK: Course and termination of the primary afferents in the cochlear nuclei of the cat: an experimental anatomical study. Arch Ital Bid 108: 21-51, 1970

Park HJ, Lee WS, Kim HN, Kim YM: Central projection of the peripheral vestibular nerve from central and peripheral parts of the lateral crista in the mongolian gerbil. Korean I Otdaryngol 35: 369-380, 1992

Rasmussen GL, Gacek RR, McCrane EP, Baker CC: Model of cochlear nucleus (cat) displaying its afferent and efferent connections. Anat Rec 136: 344, 1960

Ryan AF: Hearing sensitivity of the mongolian gerbil, Meriones unguiculatus. $J$ Acoustic $S o c$ Am 59: 1222-1226, 1976

Sando I: The anatomical interrelationship of the cochlear nuclear nerve fibers. Acta Otolaryngol 59: $417-436,1965$

Stevens SS, Davis H, Lurie MH: The localization of pitch perception on the basilar membrane. $J$ Gen Psychol 13: 297-315, 1935

Van Noort J: The anatomical basis for frequency analysis in the cochlear nuclear complex. Psychiatria Neurogia, Neurochirurgia 72: 109114, 1969

Webster DB: Projection of the cochlear nuclei in Merriam's kangaroo rat. $J$ Comp Neurol 143: 323-340, 1971 